Meta

Journal des tradlucteurs

Translators' Journal

\title{
Préface : Processus et cheminements
}

\section{Hannelore Lee-Jahnke}

Volume 50, numéro 2, avril 2005

Processus et cheminements en traduction et interprétation

Processes and Pathways in Translation and Interpretation

URI : https://id.erudit.org/iderudit/010940ar

DOI : https://doi.org/10.7202/010940ar

Aller au sommaire du numéro

Éditeur(s)

Les Presses de l'Université de Montréal

ISSN

0026-0452 (imprimé)

1492-1421 (numérique)

Découvrir la revue

Citer ce document

Lee-Jahnke, H. (2005). Préface : Processus et cheminements. Meta, 50(2),

339-341. https://doi.org/10.7202/010940ar

Ce document est protégé par la loi sur le droit d'auteur. L'utilisation des services d'Érudit (y compris la reproduction) est assujettie à sa politique d'utilisation que vous pouvez consulter en ligne.

https://apropos.erudit.org/fr/usagers/politique-dutilisation/ 


\section{Préface}

\section{Processus et cheminements}

Le traducteur a fait main basse sur trop de choses: il a rembourré, enjolivé, sollicité le texte, forcé à la lecture; ou sur trop peu: il a raboté, simplifié, arrondi les angles... pourtant l'ouvre traduite est mise en valeur et cela, à plusieurs niveaux. (Steiner 1978)

Lorsque George Steiner écrivait cet ouvrage magistral After Babel qui allait devenir une lecture incontournable en traductologie, l'étude sur les processus n'en était encore qu'à ses tout débuts. Et pourtant, le passage que nous avons cité en exergue montre, ô combien, que le cheminement du traducteur vers un produit final aussi parfait que possible était bien présent.

Depuis, de nombreuses recherches et études ont été effectuées dans le domaine des processus, que ce soit sur le plan de la traduction ou de l'interprétation. Nous en donnons pour preuve le numéro thématique de META consacré à ce sujet et qui est paru sous la direction de Frank Königs en 1996. Depuis lors, que s'est-il produit pour que André Clas décide de dédier, en cette année du 50e anniversaire de cette revue prestigieuse, un volume à cette même thématique? Au vu des contributions, je suppose que c'est l'avancement scientifique de la recherche qui peut être à l'origine de cette prise de décision. Je tiens ici à remercier André Clas du grand honneur qu'il m’a fait en me confiant la direction de ce numéro anniversaire.

Avant d'esquisser le contenu de ce numéro, j'aimerais souligner l'importance de la mise en valeur des processus notamment en didactique de la traduction. Quels sont les cheminements que nous pouvons envisager face à des situations textuelles si différentes? Comment rendre conscients, voire visibles ces processus qui nous guident, presque automatiquement? Car en présence d'un choix, il faut enseigner quel sera le chemin qui, en toute vraisemblance, sera le meilleur à suivre. Il me plaît ici de citer Maurice Pergnier (2002: 317-322) qui utilise une belle métaphore, celle de la balance: "Le succès de l'opération de la traduction dépend de la qualité de la balance ${ }^{1}$, et non de la capacité qu'ont les mots des deux langues en présence de peser in abstracto le même poids.»

Voilà autant de raisons qui justifient amplement la publication d'un numéro thématique spécial sur les processus. Comme dans chaque numéro de META, des spécialistes présentent le fruit de leurs recherches dans les domaines de la traduction et de l'interprétation. Ces recherches touchent aussi bien aux paramètres qu'aux méthodes et aspects pédagogiques.

En ce qui concerne la langue de rédaction des articles de ce numéro, nous avons, comme la fois précédente ${ }^{2}$, donné suite, en partie du moins, au vœu exprimé par André Clas dans un éditorial: «Nous aimerions en ce début de millénaire ouvrir nos pages, de temps à autre, à des articles dans une troisième langue, p. ex. l'espagnol, pour répondre ainsi aux besoins internationaux» (Clas 2000:2). C'est pourquoi, j'ai

Meta, L, 2, 2005 
ajouté à l'anglais et au français - les deux langues officielles de META - une troisième langue, l'allemand.

Ce volume comprenant 33 contributions se divise en deux grandes parties: la première, plus importante en nombre de contributions, met en relief les travaux effectués dans le domaine de la traduction et, la deuxième, ceux provenant de l'interprétation. La plupart des chercheurs ayant contribué au premier numéro de META consacré aux processus se retrouvent également dans le présent volume. L'éventail des langues et pays représentés est plus vaste qu'en 1996, ce qui est un reflet évident de l'avancement de la recherche dans notre domaine.

Le bal s'ouvre par une revue des recherches présentée par Krings, pour ensuite aborder les processus cognitifs de manière interdisciplinaire aussi bien dans le domaine de la didactique par Lee-Jahnke, de la traduction professionnelle par Kussmaul, que des protocoles de verbalisation par Rydning, ainsi que de la modélisation et du monitoring par Tirkkonen-Condit. Vandeale et Lubin présentent une systématisation de la conceptualisation métaphorique, avant d'aborder deux contributions sur la créativité, celles de Fontanet et de Heiden. Puis, arrive le grand «salto mortale de la déverbalisation » de Jean-René Ladmiral, déverbalisation qui est également l'objet des recherches de Boisson. Zhong rapporte une étude de cas effectuée en Australie et Hansen introduit l'émotion dans sa recherche qui, dans le domaine de la neurologie, a pris une importance croissante ces dernières années. Asadi et Séguinot présentent une étude réalisée avec des professionnels, et Dancette et Halimi démontrent l'importance des représentations mentales des connaissances.

Le bal continue ensuite par une partie plus «technique» avec la présentation des travaux de Masschelein/Verschueren sur l'apprentissage semi-autonome utilisant l'application Markin et Colson présente celle de TransCorrect. Eddie Ronowicz et coll. font état des résultats de leur étude comparative avec des sujets professionnels et des sujets en apprentissage conduite en Australie, et Lörscher insiste particulièrement sur les méthodes et problèmes liés au processus de traduction. Le PACTE Group, quant à lui, se penche sur la façon d'acquérir de la compétence en traduction.

Le bal se poursuit avec une autre approche des processus due à la mise en place de Bologne qui est décrite par Abou Fadel et Awaïss, avant d'aborder le e-learning développé par Massey. Balacescu et Stefanink présentent leur approche herméneutique et Gouadec modélise le processus de traduction. Wilss nous indique les facteurs de risque pouvant survenir lors des processus de traduction et, pour clore cette première partie du bal, plutôt axée vers l'avenir, une vue historique présentée par De Laet nous fait contempler le passé.

La deuxième partie du bal consacrée à l'interprétation s'ouvre avec la contribution de Pöchhacker, qui souligne l'importance des facteurs aussi bien cognitifs que conceptuels. Ahn In-kyoung analyse les causes des atteintes à la cohérence perspective des énoncés dans le couple de langue allemand-coréen, tandis que Gile poursuit ses recherches sur les aspects didactiques. Moser-Mercer met en évidence le rôle central de l'intégration multi-sensorielle en interprétation simultanée puis Mizuno, après une revue de la recherche dans le domaine ainsi que celle sur la mémoire de travail, propose un cadre théorique pour un modèle du processus de l'interprétation simultanée. Riccardi, quant à elle, souligne l'importance des stratégies et le développement d'automatismes, et Kalina propose une grille de paramètres pour assurer la qualité en interprétation. Pour terminer le bal, Benoît Kremer, après avoir décrit une 
approche méthodologique et pédagogique, introduit trois types d'exercices pratiques. C'est ainsi que contextualisation, hiérarchisation des idées et importance de la création de représentations mentales sont mises en évidence.

Le choix des sujets ainsi que des langues n'est et ne peut pas être exhaustif, mais il nous semble représentatif des recherches en cours. Ainsi, pas à pas, notre discipline, qui se cherche encore, peut trouver ses assises à l'aide de revues, telles que META, qui, par leur crédibilité, démontrent que nos disciplines langagières tiennent bel et bien une place dans le monde académique et scientifique. Un grand merci s'adresse au professeur Clas pour tout ce qu'il fait et a fait pour promouvoir la profession et placer ainsi notre discipline en bonne position dans les formations académiques.

Mes remerciements s'adressent aussi à tous les auteurs dont les contributions font la substance de ce numéro. Je saisis également l'occasion de cette préface pour exprimer ma profonde gratitude à la professeure Josiane Seydoux pour son aide précieuse et son avis critique lors de la relecture des articles effectuée avec une acribie hors pair.

HANNELORE LEE-JAHNKE

Université de Genève, Genève, Suisse

\section{NOTES}

1. Qui, pour Maurice Pergnier n'est pas dans l'ordre des mots, mais elle est plutôt faite d'idées, de sentiments et d'effets sur le lecteur.

2. Voir le numéro sur l'évaluation Lee-Jahnke (2001) 46-2.

\section{RÉFÉRENCES}

Clas, A. (2000): «Éditorial», META 45-1, p. 1-2.

KöNigs, F. (dir.) (1996): Les processus de la traduction, META 41-1, p. 5-184.

Lee-Jahnke, H. (dir.) (2001): Évaluation: Paramètres, Méthodes, Aspects pédagogiques, META 46-2.

Lee-Jahnke, H. (2002) : «Quand un poète allemand écrit en français: Rilke face à lui-même», dans: Du Pareil au même: l'auteur face à son traducteur; coll. Sources \& Cibles, Université Saint-Joseph, Beyrouth, p. 175-194.

Pergnier, M. (2002) : «Le quadrilatère conceptuel», dans: Du Pareil au même: l'auteur face à son traducteur; coll. Sources \& Cibles, Université Saint-Joseph, Beyrouth, p. 317-322.

Steiner, G. (1978): After Babel, Oxford University Press, London. 